\title{
Geropsychology: The Gender Gap in Human Aging - A Mini-Review
}

\author{
Ilse Kryspin-Exner Elisabeth Lamplmayr Anna Felnhofer \\ Institute of Clinical, Biological and Differential Psychology, Faculty of Psychology, University of Vienna, \\ Vienna, Austria
}

\section{Key Words}

Geropsychology $\cdot$ Neuropsychology $\cdot$ Aging $\cdot$ Gender $\cdot$ Sex

\begin{abstract}
Differences between women and men in terms of gender gaps at late stages of their lives are the focus of this minireview. We approach the matter by taking up the position of complex yet promising standpoints, the bio-psycho-social perspective on the one hand and approaches of developmental psychology on the other. Lifespan perspectives and the brain-behavior relationship are integrated and applied to geropsychological models. Research on gender differences is presented and examined, particularly regarding physiological and psychological differences between aging men and women; additionally, gender discrepancies in psychopathology are discussed. Prevailing concepts of 'active aging' are introduced and their implications for the review of divergent gender differences are considered.
\end{abstract}

Copyright $\odot 2011$ S. Karger AG, Basel

\section{Introduction}

In the current literature on aging-related gender differences in later life, there are different approaches to analyzing aging in general and specific gender-related differences, such as cultural historical studies as well as so- cial, structural, socioeconomic, feministic and political notions [1] and biological research [2, 3]. In our discussion of aging and gender differences, we will primarily focus on the brain-behavior relationship. While acknowledging that this is a circumscribed view of such a complex topic which may be vehemently criticized, this bridging reasonably unites biological foundations, their effects on the individual and their impact on society. This approach is in line with the bio-psycho-social model. The latter purports that throughout the entire lifespan, individuals are being confronted with multiple factors influencing their cognitive functions, physical and mental health, and duration of life $[4,5]$. With advancing age, the interaction of various factors increases in its complexity; thus, it becomes more and more difficult to apply models of linear cause and effect chains to explain gender differences.

The relevance of gender differences is reflected in an increasing number of reviews in popular science (e.g. [6, 7]) pertaining to differences in sex-specific brain structures, ways of thinking, problem solving and behavior. Accordingly, the discussion of gender differences of aging men and women has progressively awoken the interest of the public media, partly unrolling clichés and partly reinforcing preconceptions. In her recently published book, 'Why Men Die First' [8], Marianne J. Legato, who holds a clear biological perspective of gender differences, states that in general men tend to be more physiologi-

\section{KARGER \\ Fax +4161306 1234 \\ E-Mail karger@karger.ch}

www.karger.com (c) 2011 S. Karger AG, Basel

0304-324X/11/0576-0539\$38.00/0

Accessible online at:

www.karger.com/ger
Ilse Kryspin-Exner

Institute of Clinical, Biological and Differential Psychology

Faculty of Psychology, University of Vienna

Liebiggasse 5, AT-1010 Vienna (Austria)

Tel. +43 14277 47809, Fax +431 4277 47899, E-Mail ilse.kryspin-exner@univie.ac.at 
cally fragile and vulnerable than women and that this appears already around pregnancy and birth: $20 \%$ more boys than girls are conceived, and it is the male fetus that dies more often. This pattern is thought to continue throughout the whole life, with women being more resilient than men, i.e. less prone to illness and more likely to mobilize their strengths. Many of these factors are understood as protective factors for pregnancy and the upbringing of the offspring. However, as much of this evidence is equivocal, it may be speculative and problematic to generalize it to older age groups. Thus, to avoid further speculative generalizations, only 'hard' facts about sex and gender differences in older age shall be described and discussed below.

With a clear focus on a brain-behavior relationship, we divide the review of corresponding literature into 3 main domains: physiological aspects, including brain anatomy, hemisphericity and functional neuroanatomy; psychological aspects, such as cognition and emotion; and psychopathological differences.

Prior to reviewing literature and discussing recent notions in terms of aging and gender differences, light has to be shed on 4 underlying preconceptions. These include the specification of the terms 'sex' and 'gender', the conception of lifespan psychology, 'active aging' approaches, and of course sociodemographic developments, which are not only known to shape the aging process, but are also suggested to influence gender differences at later stages of life.

The labels 'sex' and 'gender' are used to separate the concepts of biological and social differences, respectively. The term 'sex' refers to biological differences among males and females based on genetic, physiological and hormonal factors. The term 'gender', however, represents social and cultural aspects of being female or male, e.g. role perception and status differences. Some authors strongly differentiate the terms 'sex' and 'gender' using sex for the distinction between men and women and gender for psychological and social constructs related to masculinity and femininity. Hines [2] argues that the distinction between sex and gender differences in humans is impossible because social and biological determination cannot be separated from each other. We concur with this position and, as we are approaching this matter from a psychological point of view on a bio-psycho-social background, we are clearly aware of the reciprocal interactions of biological and social factors. To simplify matters, we will mainly use the term 'gender' in the following text.

Aging and gender gaps are increasingly seen under the perspective of lifespan psychology [4]. Specific develop- mental tasks are taken into account and differentiated perspectives are being adopted when examining courses of life, no longer considering old age as being a distinct phase in someone's life but a developmental process that becomes operative at the time of procreation and continues until death. Developmental psychology has already contributed markedly to the understanding of developmental steps in advanced age, but the gender specific differences in late life stages have rarely been considered.

Over the last decades, a new evidence-based model accounting for a positive view on aging has been introduced to scientific gerontology. Instead of pertaining to aging as an ongoing loss of functions (as does the deficiency hypothesis), this empirically based concept highlights growth and beneficial aspects of the aging process. This affirmative view on aging adopts several terms, many of which are used interchangeably. Among healthy, successful, active, optimal and positive aging, 'active aging' is the most often mentioned term in this context [9]. The concept of 'active aging' embraces biomedical, psychological and social aspects of aging and is thus consistent with a multidimensional bio-psycho-social model, which is not to be confused with constructs of well-being or quality of life. By optimizing physical, psychological and social functioning, an 'active aging' individual may not only enhance her/his health and fitness, but may also increase cognitive abilities, emotional-motivational functioning and improve social participation.

There is empirical evidence to emphasize the relevance of the 'active aging' domains mentioned above. In the field of cognitive functioning, for instance, 'active aging' individuals who participate in many activities are predicted to perform better in cognitive tests and experience less cognitive decline over time than are those who are less active [10]. Other protective influences on psychological aspects of functioning of an active and socially integrated lifestyle shall be discussed below.

Unfortunately, gender differences or gender-specific ways of successful and healthy aging have rarely been regarded in the literature on 'active aging'. Gender, although being a crucial organizing principle in society that profoundly shapes the experience of old age and aging [11], implies a different outcome of 'active aging' for men and women. One aspect of this review will therefore be to emphasize this specific gender gap.

Our society is facing well-documented sociodemographic changes pertaining to men and women. One of the most prominent gender differences is women's longer life expectancy, a phenomenon which can be summarized quite well by the phrase: the 'feminization of old 
age' [11]. The reasons for this discrepancy in life expectancy are manifold, comprising biological and sociocultural aspects, such as differences in behavioral patterns.

Traditionally, men, for example, tend to smoke more, drink more alcohol, show riskier and more violent behavior and are exposed to more stressful and potentially debilitating factors at work than their female counterparts [4]. However, some authors claim a noticeable narrowing of this gender gap in life expectancy during the last decades. Increased smoking among women accompanied by larger increases in lung cancer mortality [12] is being discussed as one possible factor for this trend. Other authors negate this development, citing studies that demonstrate a quite stable life expectancy among women, who are exposed to the same stressful factors at work as their male coworkers [4].

Apart from an increasingly greater life expectancy among both men and women, another relevant development is taking place and can be interpreted in the sense of the 'active aging' approach. Over the last decades, the number of years spent in subjectively judged good health has increased in comparison to life expectancy in general [13]. Yet there is evidence for gender differences concerning the experience and judgment of one's own health. One study found that women at late stages of their lives tend to be more pessimistic when judging their physical state, whereas men, who are more frequently affected by life-threatening diseases than women, report better health states [4]. Another study, however, contradicted these findings, stating no differences in the self-related health ratings between men and women [14].

With these parameters in mind, we now go on to explore possible gender differences in physiology, cognition, emotion and psychopathology.

\section{Physiology}

\section{Brain and Gender Differences}

With regard to the brain-behavior relationship, one seeks to understand how the brain, through structural and neural networks, produces and controls behavior and mental processes, including emotions and cognition. Additionally the converse question of how behavior may influence the brain and related physiological processes, e.g. in the sense of enhanced cognitive abilities and increased health through 'active aging', is of great importance for geropsychological models of gender differences.

Beginning with overall age-independent anatomical differences, it has to be stated that, in general, male brains contain more neurons in specific brain areas and are therefore bigger in volume. Women, however, show a greater complexity in frontal and parietal areas, which altogether increases the cortical surface [15]. On a cellular level women seem to have more grey matter, men in turn more white substance. On the macro-structural level, corpus callosum, planum temporale and anterior cingulate cortex differ between men and women insofar as female brains are organized more symmetrically than male brains [16].

At later stages of life, aging processes, such as cortical volume reductions, have to be taken into account when investigating the neuroanatomical structure of the brain. It is then that gender differences are apparent and that impairments caused by aging processes are more likely to occur in males than in females [17]. In men, age-specific volume reductions are stronger than in females, especially in frontal and temporal lobes but also in whole brain volume. The described relations are far from definitely known, but there is evidence that brain volume reductions might lead to more deficits in executive functions in men than in women. Women also tend to show stronger reductions in the hippocampus and parietal lobe than men, possibly leading to more deficits in memory functions in women $[15,18]$. Yet again, results are inconsistent and some studies show better memory performance for women than men [19]. Brain morphology in these regions shows different patterns of age-related changes for men and women and, thus, gender differences are not constant over someone's life. It has to be noted that there are also brain regions where sex differences seem to be stable, like lateral temporoparietal regions where women have thicker cortices than men [15].

Moving away from anatomy and examining gender differences from a brain-behavior perspective, it has to be stated that there are many contradictory findings pertaining to later stages of life (for a review, see [20]). Various complex cognitive functions as well as associated disorders, which are related to functional asymmetries within the brain, show gender differences.

One hypothesis from primarily behavioral work suggests that female brains tend to be organized intrahemispherically and male brains rather bihemispherically. This means that certain functions activate multiple areas within the same hemisphere in woman or can be achieved across both hemispheres, whereas in men the alignment of function appears to be more restricted to one hemisphere than another [21]. This now seems to be supported by recent imaging results. In brain imaging studies, men show more unilateral left hemispheric activation patterns 
while solving language tasks, whereas women show bilateral activation patterns. In other words, there are hints for left lateralized language dominance in males and a more bilateral language processing in females which might protect the latter from language impairments. Taking these assumptions as a starting point, a range of studies (for a review, see [20]) has been conducted on sex differences in aphasia, stuttering and dyslexia suggesting different functioning and course of disorder for men and women. Yet, taking a closer look at the methodology of these studies, it becomes apparent that none of them can entirely sustain the hypothesis of differences between males and females in language-related cortical regions [20].

To be able to draw conclusions from the observations made on a behavioral and psychological level, functional imaging methods have been taken into account, bringing forward more knowledge of brain functions and gender. Functional differences on a cerebral level are mainly measured via functional magnetic resonance imaging (fMRI). Some fMRI studies showed consistent cerebral differences in gender [22], detecting different blood oxygen level-dependent reactions for men and women. Yet, a synopsis of multiple detailed results pertaining to gender differences at later stages of life in a geropsychological sense is still largely aspirational and has to be regarded as a 'work in progress'.

According to brain imaging studies, memory and attention deficits in aging are related to a reduction of grey matter and decreases in activation in frontal and mediotemporal areas [22]. Marguire and Frith [23] demonstrated functional differences in hippocampal areas between younger and older persons concerning autobiographic memory. Their results, indicating a left-sided activation in younger and a bilateral hippocampal activation in older subjects, led to a discussion about a possible compensation of overload in the left hippocampus in the older group. Compensation processes of hypoactivation in specific brain areas are indeed discussed by various authors in terms of aging, but not with respect to gender specificities.

Additionally, the topic of hormonal influences on brain function, especially their effects on brain lateralization [24] can be regarded as an emerging field in neuroscience, which is expected to contribute considerably to the understanding of gender differences. Thus, it shall be discussed in the following section.

\section{The Role of Hormones}

Sex hormones not only regulate sexual behavior but also influence, to a great extent, all kinds of behavior and psychological functioning, including emotion and cognition. Sex hormones interact with neural processes and with neurotransmitter systems in the brain; they directly bind to nerve cells and, thus, directly control neuronal information processing [25]. As sex hormone levels greatly change with age, it is essential to take them into account when examining possible gender gaps. Research about the effects of sex hormones on brain functions is an emerging field and the first results refer to young adults. Studies on hormonal effects on the aging brain are scarce and have to be research objectives for the near future. To stress the importance of sex hormones for neuronal functions even in older age, some basic information is reported here.

The neuroactive functions of sex hormones are divided into 2 different mechanisms: organizational effects during prenatal development and activational effects throughout life [2]. However, this distinction into 2 different mechanisms of action is not a strict one. In puberty, hormones trigger changes in brain structure as well as on the functional and behavioral level as this is a phase of major hormonal changes in both males and females. From then on women's hormones, in contrast to men's, change every month, reaching a peak during pregnancy and declining with menopause. After menopause, female sex hormone production is greatly reduced, with an accompanying impact on neuronal and psychological functioning [26].

In general, psychological actions of sex hormones may range from the relief of depressive symptoms and stress protection to mild antipsychotic effects as well as influences on cognition [26]. In the current literature on the topic, pros and cons of neuro- and psychoprotective actions of estrogens are controversially discussed with special regard to the use of hormone replacement therapy (HRT) during and after menopause. The Women's Health Initiative (WHI) Memory Study reported both positive and negative effects of HRT in menopausal women, one of their findings showing a positive effect on some cognitive functions and negative or no effects on others [26]. Subsequent studies failed to show consistently positive effects [27]. Apart from adverse reactions, these studies also found elevated risk for mild cognitive impairment and dementia in women taking HRT and, thus, HRT is not recommended for dementia prevention. At the moment it is suggested that there may be critical time windows for neuroprotective effects of postmenopausal estrogen replacement [26].

In comparison to menopause, a phenomenon called andropause has begun to be described for males, but this 
hormonal shift has not been scientifically shown to exist to a similar extent as menopause [28]. Men are confronted with hormonal changes, but these changes are not as abrupt or of as great a magnitude as hormonal changes in women. HRT with testosterone is discussed for men suffering from low serum testosterone levels [29]. Physical conditions like bone density and muscle strength might be ameliorated with testosterone medication, but mood does not seem to be affected by it as it is in women.

The way in which hormonal changes during someone's lifetime influences the developmental processes in advanced age has not been sufficiently examined, not least because of the lack of systematic longitudinal studies.

\section{Psychology}

\section{Cognitive Functions and Gender Differences}

So far, gender has played a rather subordinate role in geropsychological research on cognitive abilities. This is particularly surprising since a lot of literature points at well-established gender differences in cognition throughout life, with women generally attaining better results than men on tasks of perceptual speed and verbal fluency and men in turn excelling in tasks of visuospatial skills and mathematical reasoning [30]. Another cognitive function with strong evidence for overall gender differences is spatial cognition. In mental rotation and map reading tasks - one of the best analyzed performance tasks differentiating between women and men - men perform better on average [21]. Additionally, recent MRI studies - as mentioned in the physiology section - report greater age-related overall decline in brain volume in men than in women, which is suggested to show impact at the behavioral level. Indeed some results indicate greater age-related losses of cognitive functioning in males than in females; yet, these findings are still inconsistent, which is probably due to population biases or methodological inconsistencies.

Regarding the latter, there is a distinct lack of longitudinal observations on the same population with statistical deductions on gender differences throughout life up to older age. Besides, in functional brain imaging studies, typical participants are young to middle-aged persons; therefore, the often used term 'older' in this context does not mean old in the sense of gerontological terminology. These 2 facts should be kept in mind when conclusions about cognition and brain functions across lifespan and later stages of life are being drawn.

The Gender Gap in Human Aging
In this regard Maylor et al. [30] clearly point out the need for a suitable theoretical model to explain specific sources of gender differences in cognitive functioning both throughout life and at later stages of life. The biopsycho-social model constitutes such an approach to this matter since it embraces neurophysiological, hormonal, psychological and social factors. Additionally, paradigms of developmental psychology such as lifespan perspectives have to be incorporated into this model.

Low education as well as low levels of activity in earlier periods of life both seem to have a negative influence on later cognitive functioning [4]. Yet, the same activity may have a different impact at different stages of life and may be dependent on contextual factors like social integration, e.g. female activities in families versus single life, retirement or widowhood (the latter possibly showing variable valence for men and women) [10].

A large body of research presently aims at determining certain lifestyle habits and activities that may lead to a reduced risk of cognitive decline and dementia in old age (for a recent review, see [31]). Some authors report significant protective effects of general physical activity among all elderly people, whereas others find clear differences on both gender and the type of activity. Crowe et al. [32] in their longitudinal study on Swedish twins, for instance, attain results suggesting that engaging in intellectual-cultural activities is associated with a lower risk for Alzheimer's disease in women but not in men. Moreover, regular physical activity is reported to have protective effects on dementia risk with a higher benefit from exercise for women in particular. These findings suggest a greater importance of environmental influences on cognitive abilities in women than in men, which could be specifically due to the interaction between physical activity and hormone metabolism. Again, HRT is claimed to play an important role in protecting women from cognitive decline, but the evidence supporting this hypothesis is still quite controversial [30]. Parslow et al. [33] in turn attain contrary results compared to Crowe et al. [32]. They use a new instrument for measuring engagement in life called the RIASEC Activity List and succeed in showing that older men who engage in a greater number of activities report better physical health and perform better on cognitive tasks - the same is true only for middle-aged women. These contradicting results are depicted here to show the need for further investigation with comparable measures and concepts in order to more fully understand the interaction between activity, lifestyle and cognitive function in late life.

In spite of some results on the relationship between activity throughout life and cognitive functioning in old-

Gerontology 2011;57:539-548 
er age, we still know little about specific periods or timedependent specific domains of activities being predictors for cognitive functions in the process of aging. The restructuring of cognitive functions during the aging process in terms of 'plasticity' of the aging brain is being discussed with regard to multidimensionality, multidirectionality, multifunctionality and multicausality of cognitive functions throughout life [4]. The assumption that we possibly do not solve cognitive tasks or run through daily routines in the same way in every stage of our lives can easily be observed in the normal course of life, but is hard to demonstrate in empirical designs.

Another focus of research within the scope of aging, cognition and gender differences lies in intelligence. According to well-established theories, aging is accompanied by a sparing of crystallized abilities (e.g. vocabulary) but a worsening of fluid abilities (e.g. those processes associated with greater mental speed) [4]. However, as mentioned before, this does not predict stable effects on the behavioral level. As pointed out above, there is a greater age-related decline of overall cognitive abilities in males than in females; yet, considering mental speed per se, men seem favored. In a range of studies (cf. [30]) older men outperformed their female counterparts in mental speed tasks. This result, in comparison with results for memory, for instance, illustrates the multidimensionality of cognition and shows an area where men outperform women. However, findings for gender differences in cognition in late life are frustratingly equivocal. Data over a period of 8 years from the Australian Longitudinal Study of Ageing (ALSA [19]; $\mathrm{n}=1,947$; aged 65-105 years) demonstrated that women outperformed men in 3 cognitive domains (speed, incidental memory and verbal ability). Interestingly, rates of change did not vary by gender, but gender differences in levels of performance were partially explained by differential contributions from health, depression and education.

In a recent study about educational attainment and gender differences in fluid intelligence, crystallized intelligence and academic skills in reading, math and writing, it was demonstrated that women significantly outperformed men on the writing test and the reverse was true for the math test, whereas other gender differences were not observed to be significant [34]. According to the authors, these analyses fill a gap in the literature regarding the nature of gender and education differences in academic skills for heterogeneous samples of adults between young adulthood and old age.

As noted above, future research on cognitive functioning and gender differences at late stages of life is chal- lenged to put emphasis on one particular group of people, i.e. older adults above 80 years as did the Swiss Interdisciplinary Longitudinal Study on the Oldest Old [35]. The authors reported dynamic associations between perceptual speed and taking part in various activities. Especially engagement in media and leisure activities is correlated negatively with perceptual speed decline, yet there was no report of gender differences. Another example of a study focusing on the oldest old is BASE [4], the Berlin Aging Study, which examined older adults 70-103 years of age in a longitudinal design over a 19-year period of time and collected data concerning physiological, psychological and social aspects of development at later and last stages of life. Regarding cognitive function, the BASE sample, which consisted of 516 older adults, did not show significant gender differences. Small existing differences between men and women failed to persist after differences in education were controlled statistically [4]. By contrast and as mentioned above, the ALSA study [19] showed gender differences in the level of performance on memory, speed and language, all favoring women, even after accounting for a range of background variables, including education. It is apparent that a definitive conclusion cannot be reached about the effect of gender on cognition in late life.

\section{Emotion and Gender Differences}

Scientific research on emotions and especially on neuronal correlates of emotional processing, emotional behavior or memory of emotional stimuli has quite a short tradition. Most studies in this field mainly pertain to young and middle-aged adults and range between cliché and fact, such as the finding that women, in contrast to men, express emotions more distinctly through their behavior, or the conclusion that men have more difficulties than women in identifying sad faces or recognizing disgust and anger in faces [36]. The latter particularly addresses the issue of gender-specific socialization processes and may be especially interesting in terms of shaping interpersonal relations and social life, since recognizing emotions accurately is considered a critical factor for appropriate interpersonal functioning and higher quality of life throughout someone's life and into old age.

Yet, the current literature does not provide a definitive answer on whether emotion recognition improves, remains stable, or declines with advancing age. Moreover, research on gender differences in emotional processing or emotion recognition in older adults is scarce at best. Some efforts have been made to examine changes of predominantly facial emotion recognition in older age, with 
special regard to differences in age and variable performance across emotion types and stimulus modality but with no consideration of gender differences. Ruffman et al. [37], for instance, provide some evidence that older people find anger, fear and sadness in facial as well as in bodily expressions more difficult to recognize than young people, whereas disgust tends to be recognized more easily by older people than by younger ones. Yet, the authors fail to examine gender differences in the aging population. Gender differences in emotional processing are often observed among young adults, but are still inconclusive and need further investigation. In older cohorts, however, findings on gender differences are still rather scarce, a fact that often tends to turn hypotheses on lifetime developments into speculations.

In trying to explain these age differences, some authors favor the 'general decline hypothesis', but the fact that not all emotions are more difficult to recognize for older people contradicts this hypothesis. Therefore, the assumption of a 'more specific neuropsychological change' with frontal and temporal decline associated with more subtle changes in social behavior seems more appropriate [37].

According to brain imaging studies with younger adults, results on gender differences also vary to a great extent depending on the emotional valence of the facial expression. Happy faces, for instance, are more likely to evoke opposite activation patterns of the lateralized amygdala in men and women, with men showing an increased activation of the right amygdala and women showing an increased activation of the left amygdala. Fearful faces, in turn, induce greater amygdala activation in both men and women [38]. Again, these findings might have important implications for older people, especially with regard to the gender gap, but studies on this topic are nonexistent. As mentioned above, aging-associated changes in emotion recognition might be related to frontal and temporal decline. These frontal and temporal brain regions are tightly connected to structures inherently important for emotion, like the amygdala. Thus, gender differences might become more pronounced with age and should therefore be more closely examined in careful paradigms, i.e. narrowly defined designs examining only one particular brain area like the amygdala in fMRI with special regard to emotional valence.

A phenomenon linked conceptually to emotion recognition and facial expression processing is empathy, which has recently gained more and more attention within the neuroscience context. Baron-Cohen's theory, for instance, suggests that women are better at empathizing

The Gender Gap in Human Aging and men better at systemizing [6], but it is yet unknown if these findings can enhance the understanding of constitution versus socialization of emotion and whether they pertain to younger and older adults in the same way. Once again, there is also a lack of fMRI studies with a clear focus on older adults and gender even though empathy is undoubtedly seen as an important criterion of feminine role identity throughout life [11].

Another complex psychological phenomenon is stress, in particular life stress, which is thought to be related to microstructural changes in those brain structures that are of particular importance for information processing [39]. Again, there is no clear evidence for gender differences in aging people with regard to stress reactions. Indeed, Wang et al. [40] succeeded in demonstrating that in stress situations women tend to activate limbic structures and men neural structures associated with reasoning, but the results are not applicable to the population of older adults. It is argued that coping with stress might be different in women and men. Nevertheless, this might be an illustration that men and women, by activating different brain areas, tend to use different problem-solving strategies [3]. Women approach problems in a rather holistic manner, whereas men use a more focused manner. Altogether, older people seem to be more competent in solving everyday problems than younger people since they combine emotion regulation strategies and instrumental approaches more efficiently [41].

\section{Psychopathology}

\section{Mental Disorders, Gender and Age}

There are quite stable and reliable effects in younger age cohorts considering gender differences of prevalence rates, such as differences in the incidence and onset time of mental disorders in men and women [42].

Schizophrenia is one example for gender differences in onset time. There is a peak of illness onset in males between the ages of 15 and 25 years, whereas in women 2 peaks of onset can be observed, one at the age of about 30 years and another around menopause. In this regard, estrogen is discussed as possibly playing a protective role in preventing the disorder [43].

Substance abuse, especially alcoholism, is another basically male domain across younger samples, although the gap in the male-female ratio has decreased rapidly during the last 30 years. A possible approximation of the lifestyles of women and men - as discussed earlier - may account for these developments. In contrast to alcohol 
and substance abuse, anxiety and affective disorders show a consistently higher occurrence in women across all age cohorts, an effect which is well supported by scientific evidence [43]. The authors of the aforementioned Berlin study, BASE, succeeded to replicate these findings. In their sample, older women were more likely to show anxiety and depressive symptoms than men, and the effect persisted even when domestic circumstances and marital status were controlled [4]. Data from ALSA also support these trends [19].

When attempting to explain these differences within brain-behavior models, mood changes and mood disorders are related to changes in stress response and the hypothalamic-pituitary-adrenal (HPA) system. Sex hormones, in turn, are held responsible for influencing the HPA system. As sex hormones and especially their relative levels change dramatically with menopause, this might be a trigger for mental disorders in women in that period [44] conjointly going along with social and psychological changes (e.g. significant life events such as the so-called 'empty nest' syndrome when children leave their parental homes, divorce, taking care for aged parents and others).

Alzheimer's disease is another example for higher prevalence rates in women [4]. However, when women's higher life expectancy and survival rates after diagnosis are taken into account, it is not gender but age that seems to constitute the primary risk factor for dementia. Yet, the relationship between age and the gender-dependent prevalence rate is still puzzling [4]. As mentioned earlier, there is evidence for and also against neuroprotective effects of estrogens and, thus, HRT in women is discussed controversially as being a possible protection from cognitive decline (see above and [17]).

For another type of dementia, vascular dementia, higher prevalence in men than in women as well as equal prevalence rates are reported [45]. As the vascular condition is a risk factor for vascular dementia, it seems plausible that gender differences in vascular dementia prevalence decrease because of the distribution of vascular risk factors being increasingly equal between men and women due to changing lifestyle factors.

\section{Summary and Discussion}

When reviewing the literature with its inconsistent and partially contradictory findings, it becomes clear that combining developmental and biological interpretations with regard to aging and gender differences is cer- tainly of great interest. Yet, an undifferentiated consideration of this subject may lead to reductionistic interpretations. Thus, any attempt to come to terms with the aging-related gender differences needs to consider the following factors which cannot be separated from one another as they are enmeshed: specific and/ or unspecific genetic factors, gender-specific organization of the brain structures with diverse maturation processes or sex-specific neuronal maturation rates, influences of sex hormones, environmental influences, learning experiences, and gender-specific cognitive strategies. With increasing age, these factors underlie a more complex interaction with parameters like lifestyle, education, role models, autobiography, changing social networks and an individual's value system, to name a few.

It is apparent that findings about gender differences in psychological functioning, such as cognition and emotion of young people in reproductive age cannot be extrapolated one-to-one to older people (e.g. women in the postreproductive phase). Results attained from a sample of young-to-middle-aged adults do not necessarily pertain to older adults. They may result in contradictory assumptions, and mean scores observed in younger adults may no longer apply to the diversity seen in older adults' performance. For instance, physiological gender discrepancies of brain anatomy and physiological functioning remain a puzzle among the oldest old. Furthermore, gender differences in cognitive strategies raise the hypothesis that problem solving might be different in men and women because of different neuronal networks being activated; therefore, inferences on age-related gender differences have to be undertaken with great care. Emerging research of more complex functions, such as empathy and coping with stress, seems promising, foremost because of its objective to discover gender specifications throughout someone's lifetime that would support strategies for better aging in terms of social and cognitive functioning. These analyses could also be a contribution to older women's and men's capacities to stay healthy, participate in social life and maintain personal safety (see 'gender mainstreaming' in older age [11]). According to the engagement theory, there is a lot of empirical evidence that 'active aging' is one vehicle to support this. Both biological factors and substantially different cohort-dependent gender roles, which consequently influence the types of activities men and women participate in, may equally have an impact on physical condition and cognition in older age. Yet, as reported in this review, there is still little knowledge about these processes, and if aging is to be perceived positively and optimistically, and not in the 
sense of an ongoing loss of functions, they have to be analyzed more precisely with particular attention and care to methodological designs.

\section{Conclusion}

Several themes emerged in the course of this review. First, when speaking of gender differences in general, it has to be kept in mind that gender as a variable is clearly correlated with demographic factors and might therefore be regarded as a 'proxy variable' that still has to be specified to a greater extent. Second, as in any cross-sectional design, when regarding gender discrepancies among older adults, one has to pay attention to the possibility that age and cohort may be confounded. The fact that different cohorts were born and raised in diverse sociohistori- cal periods renders findings of cross-sectional studies questionable in regard to identifying underlying developmental processes. Finally, cross-sectional designs per se introduce a lot of inconsistencies into scientific findings of gender differences in old age. This is due to the fact that only with longitudinal designs can one establish sophisticated hypotheses about causal inferences pertaining to individuals and groups.

In conclusion, the combination of developmental and biological interpretations adds to an enlarged understanding of gender-specific aging. Relating these considerations to evolutionary approaches as is quite often done in recent research is certainly of great interest - yet, discussing this matter on a superficial level bears the risk of creating oversimplified attitudes, which women have long sought to overcome.

\section{References}

1 Arber S: Gender and Ageing Changing Roles and Relationships. Maidenhead, Open University Press, 2003.

2 Hines M: Brain Gender. New York, Oxford University Press, 2004.

3 Lautenbacher S, Güntürkün O, Hausmann M: Gehirn und Geschlecht - Neurowissenschaft des kleinen Unterschieds zwischen Frau und Mann; mit 24 Tabellen. Heidelberg, Springer, 2007.

4 Lindenberger U, Smith J, Mayer K, Baltes P (eds): Die Berliner Altersstudie, ed 3. Berlin, Akademie Verlag, 2010.

5 Baltes P, Mayer K: The Berlin Aging Study: Aging from 70 to 100 . New York, Cambridge University Press, 1999.

6 Baron-Cohen S: The Essential Difference. Men, Women and the Extreme Male Brain. Allen Lane, Penguin Books, 2003.

7 Brizendine L: The Female Brain, ed 1. New York, Morgan Road Books, 2006.

8 Legato MJ: Why Men Die First. New York, Palgrave Macmillan, 2008.

9 Fernandez-Ballesteros R: Active Aging: The Contribution of Psychology. Göttinger, Hogrefe, 2008.

10 Salthouse TA: Mental exercise and mental aging. Perspect Psychol Sci 2006;1:68-87.

- 11 Perrig-Chiello P, Hutchison S: Health and well-being in old age: the pertinence of a gender mainstreaming approach in research. Gerontology 2010;56:208-213.

12 Bobak M: Relative and absolute gender gap in all-cause mortality in Europe and the contribution of smoking. Eur J Epidemiol 2003; 18:15-18.
13 Kocka J, Staudinger UM (eds): Gewonnene Jahre. Empfehlungen der Akademiegruppe Altern in Deutschland. Stuttgart, Wissenschaftliche Verlagsgesellschaft, 2009, vol 9, p 80.

14 Sargent-Cox KA, Anstey KJ, Luszcz MA: Determinants of self-rated health items with different points of reference: implications for health measurement of older adults. J Aging Health 2008;20:739-761.

15 Sowell ER, Peterson BS, Kan E, Woods RP, Yoshii J, Bansal R, Xu D, Zhu H, Thompson PM, Toga AW: Sex differences in cortical thickness mapped in 176 healthy individuals between 7 and 87 years of age. Cereb Cortex 2007;17:1550-1560.

16 de Courten-Myers GM: The human cerebral cortex: gender differences in structure and function. J Neuropathol Exp Neurol 1999;58: 217-226.

-17 Azad NA, Al Bugami M, Loy-English I: Gender differences in dementia risk factors. Gend Med 2007;4:120-129.

18 Nieuwenhuys R, Voogd J, Huijzen C: Hypothalamus; in Nieuwenhuys R, Voogd J, Huijzen C (eds): The Human Central Nervous System. Berlin, Springer, 2008, pp 314320.

19 Luszcz M, Giles L, Eckermann S, Edwards P, Browne-Yung K, Hayles C: The Australian Longitudinal Study of Ageing: 15 years of ageing in South Australia. SADoFa, http:// flinders.edu.au/sabs/fcas/alsa, 2007.

20 Wallentin M: Putative sex differences in verbal abilities and language cortex: a critical review. Brain Lang 2009;108:175-183.

21 Kimura D: Sex differences in the brain. Sci Am 1992;267:118-125.
22 Reske M, Habel U: Geschlechts- und altersabhängige Effekte; in Schneider F, Fink G (eds): Funktionelle MRT in Psychiatrie und Neurologie. Berlin, Springer, 2007, pp 177185.

23 Maguire EA, Frith CD: Aging affects the engagement of the hippocampus during autobiographical memory retrieval. Brain 2003; 126:1511-1523.

24 Hausmann M, Gunturkun O: Steroid fluctuations modify functional cerebral asymmetries: the hypothesis of progesterone-mediated interhemispheric decoupling. Neuropsychologia 2000;38:1362-1374.

-25 Birzniece V, Backstrom T, Johansson IM, Lindblad C, Lundgren P, Lofgren M, Olsson T, Ragagnin G, Taube M, Turkmen S, Wahlstrom G, Wang MD, Wihlback AC, Zhu D: Neuroactive steroid effects on cognitive functions with a focus on the serotonin and GABA systems. Brain Res Rev 2006;51:212239.

26 Sherwin BB: Estrogen and cognitive aging in women. Neuroscience 2006;138:1021-1026.

27 Petitti DB, Crooks VC, Chiu V, Buckwalter JG, Chui HC: Incidence of dementia in longterm hormone users. Am J Epidemiol 2008; 167:692-700.

28 Seidman SN: Normative hypogonadism and depression: does 'andropause' exist? Int J Impot Res 2006;18:415-422.

-29 Perry PJ, Lund BC, Arndt S, Holman T, Bever-Stille KA, Paulsen J, Demers LM: Bioavailable testosterone as a correlate of cognition, psychological status, quality of life, and sexual function in aging males: implications for testosterone replacement therapy. Ann Clin Psychiatry 2001;13:75-80. 
30 Maylor EA, Reimers S, Choi J, Collaer ML, Peters M, Silverman I: Gender and sexual orientation differences in cognition across adulthood: age is kinder to women than to men regardless of sexual orientation. Arch Sex Behav 2007;36:235-249.

- 31 Bielak AAM: How can we not 'lose it' if we still don't understand how to 'use it'? Unanswered questions about the influence of activity participation on cognitive performance in older age - a mini-review. Gerontology 2010;56:507-519.

- 32 Crowe M, Andel R, Pedersen NL, Johansson B, Gatz M: Does participation in leisure activities lead to reduced risk of Alzheimer's disease? A prospective study of Swedish twins. J Gerontol B Psychol Sci Soc Sci 2003; 58:249-255.

33 Parslow RA, Jorm AF, Christensen H, Mackinnon A: An instrument to measure engagement in life: factor analysis and associations with sociodemographic, health and cognition measures. Gerontology 2006;52:188198.

34 Kaufman AS, Kaufman JC, Liu X, Johnson CK: How do educational attainment and gender relate to fluid intelligence, crystallized intelligence, and academic skills at ages 22-90 years? Arch Clin Neuropsychol 2009; 24:153-163.
35 Ghisletta P, Bickel JF, Lovden M: Does activity engagement protect against cognitive decline in old age? Methodological and analytical considerations. J Gerontol B Psychol Sci Soc Sci 2006;61:253-261.

-36 Montagne B, Kessels RPC, Frigerio E, de Haan EHF, Perrett DI: Sex differences in the perception of affective facial expressions: do men really lack emotional sensitivity? Cogn Process 2005;6:136-141.

37 Ruffman T, Henry JD, Livingstone V, Phillips LH: A meta-analytic review of emotion recognition and aging: implications for neuropsychological models of aging. Neurosci Biobehav Rev 2008;32:863-881.

38 Wager TD, Phan KL, Liberzon I, Taylor SF: Valence, gender, and lateralization of functional brain anatomy in emotion: a metaanalysis of findings from neuroimaging. Neuroimage 2003;19:513-531.

39 Seckfort DL, Paul R, Grieve SM, Vandenberg B, Bryant RA, Williams LM, Clark CR, Cohen RA, Bruce S, Gordon E: Early life stress on brain structure and function across the lifespan: a preliminary study. Brain Imaging Behav 2008;2:49-58.
40 Wang JL, Lesage A, Schmitz N, Drapeau A: The relationship between work stress and mental disorders in men and women: findings from a population-based study. J Epidemiol Community Health 2008;62:42-47.

41 Fredda B-F: Everyday problem solving and emotion: an adult developmental perspective. Curr Dir Psychol Sci 2007;16:26-31.

42 Jacobi F, Wittchen HU, Holting C, Hofler M, Pfister H, Muller N, Lieb R: Prevalence, comorbidity and correlates of mental disorders in the general population: results from the German Health Interview and Examination Survey (GHS). Psychol Med 2004;34:597611.

43 Riecher-Rossler A, de Geyter C: The forthcoming role of treatment with oestrogens in mental health. Swiss Med Wkly 2007;137: 565-572.

44 Swaab DF, Bao AM, Lucassen PJ: The stress system in the human brain in depression and neurodegeneration. Ageing Res Rev 2005;4: 141-194.

-45 Sachdev PS, Brodaty H, Valenzuela MJ, Lorentz L, Looi JCL, Berman K, Ross A, Wen W, Zagami AS: Clinical determinants of dementia and mild cognitive impairment following ischaemic stroke: The Sydney Stroke Study. Dement Geriatr Cogn Disord 2006;21: 275-283. 